\title{
Aerial assessment of landscape net change by means of two-phase network sampling: an application to central Italy
}

\author{
Piermaria Corona ${ }^{1}$, Lorenzo Fattorini ${ }^{2 * \dagger}$ and Enrico Pompei ${ }^{1}$ \\ ${ }^{1}$ Dipartimento di Scienze dell'Ambiente Forestale e delle sue Risorse, Università della Tuscia, Viterbo, Italy \\ ${ }^{2}$ Dipartimento di Metodi Quantitativi, Università di Siena, Siena, Italy
}

\begin{abstract}
SUMMARY
A design-based procedure for estimating the land cover net change between two dates in time is considered by means of aerial information. The aerial information in the first date consists of analogical photos while the aerial information in the second date consists of digital photos or satellite imagery. In the first phase of sampling a set of points is selected on the study area by means of unaligned systematic sampling. The digital imagery available for the second date may be readily adopted for spotting and classifying the first-phase points, thus obtaining a firstphase estimator of the areal extents in the second date. On the other hand, owing to the operational difficulties of spotting all the first-phase points on the analogical photos, a second-phase sample selected from these points is necessary to estimate the areal extents of the land categories in the first date. Finally, the land cover net changes may be estimated by means of the difference between the resulting estimates in the two dates. The use of stratified network sampling is considered in the second phase in order to handle the presence of analogical photos overlapping each other at their boundaries. An application of the procedure is considered for assessing the land cover net change in the Abruzzo Region (Central Italy) between the years 1954 and 2002. Copyright (C) 2006 John Wiley \& Sons, Ltd.
\end{abstract}

KEY WORDS: land cover net change; design-based estimation; two-phase sampling; unaligned systematic sampling; stratified network sampling

\section{INTRODUCTION}

In temperate countries, landscapes are far from static. Human management interacts with natural disturbance regimes and semi-natural perturbations in a dynamic process to shape the present landscape composition and configuration. A major effort is needed to manage such landscape dynamics and to try to make this type of change economically and ecologically viable by suitable mitigation and compensation planning. In light of this, the objective assessment of historical land cover evolution is essential to design adaptive planning strategies, for instance under an ecological landscape perspective (e.g., Dale et al., 2000).

*Correspondence to: L. Fattorini, Dipartimento di Metodi Quantitativi, P.za S. Francesco 8, 53100 Siena, Italy.

${ }^{\dagger}$ E-mail: fattorini@unisi.it 